(1)

\title{
O uso de dispositivos auxiliares para marcha em idosos e sua relação com autoeficácia para quedas
}

\author{
Vanessa S. Albuquerque, ${ }^{1}$ Laíze P. Fernandes, ${ }^{2}$ Francisco Eduardo F. Delgado, ${ }^{3}$ Cláudia Helena C. Mármora ${ }^{2^{*}}$
}

\section{Resumo}

Introdução: O aumento da longevidade impõe cada vez mais a necessidade de estudos relacionados à promoção do envelhecimento funcional e com maior independência e autonomia. Neste contexto, a utilização dos dispositivos auxiliares para a marcha visando prevenção de quedas e a melhora da qualidade de vida nessa população pode ser positiva aumentando a autoeficácia para queda, como também interferir de forma negativa aumentando o risco de quedas. O objetivo deste estudo foi verificar se existe relação entre o uso de dispositivos auxiliares para marcha no aumento da autoeficácia para quedas nos idosos participantes. Método: Trata-se de um estudo descritivo com dados do Banco FIBRA - JF com a utilização das variáveis de interesse para os objetivos deste estudo. A amostra foi composta por 280 idosos, sendo verificado o uso de dispositivos auxiliares para a marcha e as respostas da Escala de autoeficácia para quedas (FES-I). Resultados: A relação da autoeficácia com o uso de dispositivos auxiliares foi significativa nas questões que dizem respeito a subir ou descer escadas, andar sobre superfície escorregadia e subir e descer uma ladeira. Os resultados do grupo sem dispositivo auxiliar de marcha (GSDAM), foram significativos quanto à autoeficácia nos itens relativos às atividades externas e sociais. Ao compararmos a autoeficácia com o evento de quedas no GSDAM, foi verificada uma relação direta entre os indivíduos que se mostraram extremamente preocupados em cair e os que de fato caíram. Conclusão: O uso de dispositivos auxiliares para a marcha não aumenta efetivamente a autoeficácia para quedas em idosos, e tais dispositivos não podem ser considerados instrumentos únicos e confiáveis na prevenção de quedas.

Descritores: Bengala; Equipamentos assistivos; Fisioterapia; Acidentes por quedas; Idoso frágil.

\footnotetext{
Abstract

The use of auxiliary devices for marching in elderly and their relationship with self-efficacy for falls Introduction: The increase in longevity demands studies on the promotion of functional aging with greater independence and autonomy. In this context, the use of assistive devices (DAM) for gait to prevent falls and improvement the quality of life in this population can be positive, increasing the self-efficacy for fall, but also interfering in a negative way increasing the risk of falls. The objective of this study was to verify if there is a relationship between the use of gait auxiliary devices in increasing self-efficacy for falls in the elderly participants. Method: This is a descriptive study with data from Banco FIBRA-JF with the use of variables of interest. The sample
}

\footnotetext{
1. Faculdade de Fisioterapia. Programa de Mestrado em Ciências da Reabilitação. Universidade Federal de Juiz de Fora. Juiz de Fora, MG, Brasil.

2. Faculdade de Fisioterapia. Universidade Federal de Juiz de Fora. Juiz de Fora, MG, Brasil.

3. Faculdade de Ciências da Saúde. Universidade Presidente Antônio Carlos - Campus VI. Juiz de Fora, MG, Brasil.
}

*Endereço para correspondência:

Faculdade de Fisioterapia, UFJF.

Avenida Eugênio do Nascimento s/no

Juiz de Fora, MG, Brasil. CEP: 36038-330.

E-mail: claudia.marmora@ufjf.edu.br

Revista HUPE, Rio de Janeiro, 2018;17(2):51-56

doi: $10.12957 /$ rhupe.2018.40858

Recebido em 07/05/2018. Aprovado em 23/11/2018.

consisted of 280 elderly people, with the use of gaiters and the Self-Efficacy Scale (FES-I) responses. Results: The relationship between self-efficacy and the use of assistive devices was significant in questions concerning climbing or descending stairs, walking on slippery surfaces and climbing up and down a slope. The results of the group without auxiliary gait device (GSDAM) were significant for self-efficacy in the items related to external and social activities. When comparing self-efficacy with the fall event in the GSDAM, a direct relationship was found between individuals who were extremely concerned about falling and those who actually fell. Conclusion: The use of walking aids does not effectively increase self-efficacy for falls in the elderly and cannot be considered as a reliable single tool for falls prevention.

Keywords: Cane; Assistive devices; Physiotherapy; Accidents involving falls; Fragile elderly.

\section{Resumen}

El uso de dispositivos auxiliares para marcha en idosos y su relación con autoeficacia para quedas

Introducción: El aumento de la longevidad impone cada vez más la necesidad de estudios relacionados con la promoción del envejecimiento funcional y con mayor independencia y autonomía. En este contexto, la utilización de los dispositivos auxiliares para la marcha para la prevención de caídas y la mejora de la calidad de vida en esa población puede ser positiva aumentando la auto-eficacia para la caída, como también 


\section{Artigo original}

interferir de forma negativa aumentando el riesgo de caídas. El objetivo de este estudio fue verificar si existe relación entre el uso de dispositivos auxiliares para marcha en el aumento de la autoeficacia para caídas en los ancianos participantes. Método: Se trata de un estudio descriptivo con datos del Banco FIBRA - JF con la utilización de las variables de interés para los objetivos de este estudio. La muestra fue compuesta por 280 ancianos siendo comprobado el uso de dispositivos auxiliares para la marcha y las respuestas de la Escala de autoeficacia para caídas (FES-I). Resultados: La relación entre auto-eficacia con el uso de dispositivos auxiliares fue significativa en las cuestiones que se refieren a subir o bajar escaleras, andar sobre superficie resbaladiza y subir y bajar una pendiente. Los resultados del

\section{Introdução}

$\mathrm{O}$ aumento da longevidade trouxe mudanças no modo de pensar e viver a velhice em todas as dimensões da vida humana. Tais mudanças têm favorecido a manutenção da autonomia e independência das pessoas idosas. Por meio de um protagonismo social elas têm buscado a promoção do seu próprio envelhecimento funcional, impondo cada vez mais a necessidade de estudos e pesquisas neste campo. ${ }^{1}$

A mobilidade é um aspecto essencial para a independência nas atividades de vida diária. $\mathrm{Na}$ população idosa, é comum encontrarmos distúrbios relacionadosà marcha quenemsempresãosolucionados por tratamentos clínicos ou cirúrgicos, sendo na maioria das vezes necessário algum tipo de assistência externa para que essa população deambule com segurança. ${ }^{2}$ Os dispositivos auxiliares de marcha (DAM) podem ajudar a reduzir a carga dos membros inferiores e assim, aliviar dores articulares ou compensar fraquezas e lesões, contribuindo para o aumento da independência funcional e a estabilidade postural. ${ }^{3}$

A utilização desses auxiliares de marcha pode ser muito vantajosa para adultos mais velhos quando prescritos corretamente. ${ }^{3,4}$ A bengala e o andador são os DAM mais utilizados e sua prescrição deve levar em conta a capacidade cognitiva, visual e vestibular, a força dos membros superiores, a resistência muscular, o condicionamento físico e as demandas ambientais para determinar se há condições para tal uso.

As bengalas são, em geral, prescritas para pacientes com níveis moderados de deficiência e mobilidade, devendo ser utilizadas homolateralmente ao membro inferior mais funcional. Já os andadores, são prescritos para idosos com fraqueza generalizada, com deficit de equilíbrio e para aqueles que necessitam de diminuição de descarga ${ }^{1}$ de peso nos membros inferiores. ${ }^{1-3}$ grupo sin dispositivo auxiliar de marcha (GSDAM), cuando no hicieron uso de auxiliares para la marcha con la auto-eficacia de esos pacientes fue significativo en los ítems relativos a las actividades externas y sociales. Al comparar la auto-eficacia con el evento de caídas en el GSDAM, se verificó una relación directa entre los individuos que se mostraron extremadamente preocupados en caer y los que de hecho cayeron. Conclusión: El uso de dispositivos auxiliares para la marcha no aumenta efectivamente la auto-eficacia para caídas en ancianos y no pueden ser considerados instrumentos únicos y confiables en la prevención de caídas.

Palabras clave: Bengala; Equipos asistivos; Fisioterapia; Accidentes por caídas; Ancianos fragilizados.

Embora diversos estudos tenham demonstrado que os DAM são eficazes na melhora do equilíbrio e da mobilidade por meio do aumento da estabilidade e da redução da carga de peso nas extremidades inferiores, outros sugerem que quando utilizados de forma incorreta eles podem contribuir para uma locomoção insegura e aumentar o risco de quedas. ${ }^{3-5}$

A autoeficácia é vista como um conjunto perceptivo de habilidades do indivíduo ou autoconfiança em lidar com uma determinada situação. ${ }^{8}$ Dessa maneira, a baixa autoeficácia para quedas pode ser relacionada ao medo de cair, o que muitas vezes leva ao indivíduo a evitar realizar algumas atividades, fato este que contribui ainda mais para o declínio funcional decorrente de inatividade e aumento do risco de quedas. . $^{8-10}$

Sendo assim, o objetivo deste estudo foi verificar se existe relação entre o uso de dispositivos auxiliares para marcha e o aumento da autoeficácia para quedas nos idosos participantes.

\section{Método}

Este estudo foi desenvolvido a partir do banco do banco de dados do projeto multicêntrico rede FIBRA Brasil de delineamento transversal e analítico realizado pelo polo UERJ com a população idosa do município de Juiz de Fora. ${ }^{11}$ A pesquisa foi realizada com respeito a todos os padrões éticos aprovados pela Comissão Nacional de Ética em Pesquisa do Ministério da Saúde (CONEPE/MS - 313/2008) sob o processo número 555087/2006-9 com o apoio do Conselho Nacional de Desenvolvimento Científico e Tecnológico (CNPq). A coleta de dados ocorreu nos anos 2008-2009, nas residências dos idosos e foi realizada por pesquisadores da Universidade Federal de Juiz de Fora devidamente treinados. ${ }^{11}$ 
Os dados do questionário FIBRA-JF analisados no presente estudo foram os seguintes: 1 . as perguntas sobre o uso de dispositivos auxiliares para a marcha: "186.a. O(A) Sr./Sra. habitualmente usa algum auxiliar de marcha, como bengala ou andador?"; 2. os itens da Escala de autoeficácia para quedas, Falls Eficacy Scale International (FES-I), desenvolvida por Yardley e validada por Camargos no Brasil,que avalia a preocupação com a possibilidade de cair ao realizar 16 atividades de vida diária, com respectivos escores de um a quatro variando de 16 (ausência de preocupação) a 64 (preocupação extrema) e 3. o histórico de quedas com a seguinte pergunta: "77: No último ano o(a) Sr/ Sra. sofreu alguma queda?"12,13

A amostra selecionada deste estudo avaliou ( $\mathrm{n}=278)$ indivíduos do Banco FIBRA-JF. A partir desta seleção a mesma foi dividida em 2 subgrupos, sendo o primeiro denominado 'Grupo Dispositivo Auxiliar de Marcha' (GDAM) (n=253) composto por indivíduos que não utilizam dispositivos auxiliares, tais como bengala e andador. O segundo grupo $(n=25)$ foi composto por indivíduos que não fazem uso dos dispositivos, denominado ‘Grupo Sem Dispositivo' (GSDAM).

\section{Análise dos dados}

Primeiramente foi feita uma análise descritiva das variáveis de interesse anteriormente citadas analisando média, desvio padrão, mediana. Em seguida foi feita uma correlação utilizando o teste $X^{2}$ de Pearson por meio da análise de dois grupos, onde se verificou o aumento ou diminuição da autoeficácia para quedas nos idosos que fazem ou não o uso de dispositivos auxiliares de marcha e como a amostra do estudo reagiu ao uso de dispositivos em relação ao número de quedas (<2 quedas/nos últimos 12 meses).

A análise das informações colhidas foi feita inicialmente no programa estatístico Statistical Package for the Social Sciences (SPSS) versão 20.0. Para a análise posterior foi construído um banco de dados pela tabulação e codificação dos dados que foram analisados pelo programa Sphinx Lexica \& Eureca v. 5, estabelecendo a frequência de cada variável na amostra levantada. O nível de significância considerado foi de 0,05 para todas as análises.

\section{Resultados}

A média de idade da população foi de 75,73 \pm 7,01 anos e a maioria dos indivíduos não utilizava dispositivos auxiliares para marcha.

Relacionando os dados sobre a autoeficácia para quedas (FES-I) com a utilização ou não dos dispositivos auxiliares para marcha, verificou-se que não houve diferença estatística entre os grupos $(p>0,05)$. Em relação ao evento quedas em cada grupo $44 \%$ dos idosos que usam dispositivos sofreram quedas e 34\% dos que não utilizavam dispositivos auxiliares para a marcha também sofreram quedas (TABELA 1).

$\mathrm{Na}$ análise dos itens isolados de cada um dos grupos, foram encontrados resultados significativos $(\mathrm{p}<0,05)$ no GDAM quando comparadas as questões da FES-I que dizem respeito aos seguintes itens: subir ou descer escadas ( $n^{\circ} 193$ ), andar sobre superfície escorregadia ( $n^{\circ}$ 197), caminhar sobre superfície irregular ( $\left.n^{\circ} 200\right)$ e subir e descer uma ladeira ( $\left.n^{\circ} 201\right)$ (TABELA 2). Nesses casos, os valores representam pouco preocupado, muito preocupado e extremamente preocupado, inferindo-se que a preocupação em cair se refere ao tipo de terreno que o indivíduo encontra e suas características.

Tabela 1. Relação entre idosos que usam e os que não usam DAM com quedas

\begin{tabular}{lcccc}
\hline & \multicolumn{2}{c}{ Não usam } & \multicolumn{2}{c}{ Usam } \\
& no & $\%$ & no & $\%$ \\
\hline Sim & 85 & 34 & 11 & 44 \\
Não & 168 & 66 & 14 & 56 \\
Não sabe & 0 & 0 & 0 & 0 \\
Não respondeu & 0 & 0 & 0 & 0 \\
\hline Total & 263 & 100 & 25 & 100 \\
\hline
\end{tabular}

Produzido pelos autores. *DAM: dispositivo auxiliar de marcha, $\mathrm{N}=$ número de idosos e frequência dos participantes $\%=$ porcentagem.

Tabela 2. Autoeficácia nos itens isolados da FES-I no GDAM

\begin{tabular}{ccc}
\hline Questões da FES-I & Quesito de significância & Valor de $p$ \\
\hline no 193 & Um pouco preocupado & $<0,01$ \\
nㅇ 197 & Muito preocupado & $<0,01$ \\
nㅇ 200 & Extremamente preocupado & $<0,01$ \\
nㅇ 201 & Muito preocupado & $<0,01$ \\
\hline
\end{tabular}

Produzido pelos autores. Nota: 193 - Subir ou descer escadas; *197 - Caminhando sobre superfícies escorregadias; $* 200-$ Caminhando sobre superfícies irregulares e 201 - Subindo ou descendo ladeiras. 


\section{Artigo original}

Tabela 3. Autoeficácia nos itens isolados da FES-I no GDAM

\begin{tabular}{|c|c|c|c|}
\hline Questões da FES-I & Quesito de significância & Quedas & Valor de $p$ \\
\hline & Nem um pouco preocupado & NÃO & \\
\hline \multirow[t]{3}{*}{ no 187} & & & $<0,03$ \\
\hline & Um pouco preocupado & SIM & \\
\hline & Muito preocupado & SIM & \\
\hline \multirow[t]{2}{*}{ no 194} & & & $<0,042$ \\
\hline & Extremamente preocupado & SIM & \\
\hline
\end{tabular}

Produzido pelos autores. Nota: *187 - Limpando a casa e *194 - Caminhando pela vizinhança.

Tabela 4. Auto-eficácia nos itens isolados da FES-I no Grupo GSDAM

\begin{tabular}{|c|c|c|}
\hline Questões da FES-I & Quesito de significância & Valor de $p$ \\
\hline no 187 & Extremamente preocupado & $<0,01$ \\
\hline no 188 & Extremamente preocupado & $<0,01$ \\
\hline no 191 & Um pouco preocupado & $<0,01$ \\
\hline no 192 & Muito preocupado & $<0,01$ \\
\hline no 194 & Um pouco preocupado & $<0,01$ \\
\hline no 196 & Um pouco preocupado & $<0,01$ \\
\hline no 197 & Muito preocupado & $<0,01$ \\
\hline no 200 & Um pouco preocupado & $<0,01$ \\
\hline no 202 & Muito preocupado & $<0,01$ \\
\hline
\end{tabular}

Produzido pelos autores. Nota: ${ }^{*} 187$ - Limpar a casa; $* 188$ - Vestir ou tirar a roupa; *191 - Indo às compras; *192 - Sentar ou levantar de uma cadeira; *194 - Caminhar pela vizinhança; *196 - Ir atender ao telefone antes que ele pare de tocar; *197 - Caminhar sobre superfície escorregadia; *200 - Caminhar sobre superfícies irregulares e *202 - Ir a uma atividade social.

Ainda nesse grupo, quando analisado o evento quedas e os resultados dos itens da FES-I, foi encontrada relevância apenas nas seguintes questões: "187. Limpar a casa" e "194. Caminhar pela vizinhança" (TABELA 3).

Quanto aos indivíduos do grupo GSDAM e independentes para a marcha, a análise de alguns itens da FES-I mostrou resultados altamente significativos quando não há presença do dispositivo auxiliar de marcha nos seguintes itens: limpar a casa $\left(\mathrm{n}^{\mathrm{0}} 187\right)$, vestir ou tirar a roupa ( $\left.\mathrm{n}^{\mathrm{o}} 188\right)$, indo às compras $\left(\mathrm{n}^{\mathrm{o}}\right.$ 191), sentando ou levantando de uma cadeira $\left(\mathrm{n}^{\circ}\right.$ 192), caminhando pela vizinhança $\left(\mathrm{n}^{\mathrm{N}} 194\right)$, ir atender ao telefone antes que pare de tocar ( $\left.\mathrm{n}^{\mathrm{o}} 196\right)$, andar sobre superfície escorregadia ( $\left.\mathrm{n}^{\mathrm{0}} 197\right)$, caminhar sobre superfície irregular ( $\mathrm{n}^{\circ} 200$ ) e ir a uma atividade social (nº2). Nestes, os valores que apontam relevância nos quesitos são: pouco preocupado, muito preocupado e extremamente preocupado (TABELA 4).

No grupo GSDAM a comparação dos resultados entre autoeficácia e evento de quedas mostrou uma relação direta entre os indivíduos que se mostraram extremamente preocupados em cair e os que de fato caíram (TABELA 5).

\section{Conclusão}

Este estudo mostra resultados que comparam a autoeficácia nos dois grupos indicando que o uso de dispositivos auxiliares não interferiu na preocupação dos indivíduos em cair. Nesta direção, outros estudos indicam que o medo de cair aumenta de acordo com a idade e tem sido relatado tanto por idosos que já sofreram um evento de queda como por aqueles que nunca caíram. Neste sentido, o medo de cair encontrase relacionado à redução do nível de atividades, conduzindo a um declínio funcional, inatividade e aumento do risco de quedas. ${ }^{14-16}$

Na população idosa, a perda da autoeficácia ou autoconfiança em lidar com uma determinada situação pode estar relacionada também a fatores ligados a fragilidade, declínio funcional e perturbação do controle postural. ${ }^{17,18}$ Nosso estudo aponta que o grupo que não utiliza nenhum auxiliar de marcha teve baixa autoeficácia 
Tabela 5. Autoeficácia relacionada ao evento quedas no GSDAM

\begin{tabular}{cccc}
\hline Questões da FES-I & Quesito de significância & Quedas & Valor de $p$ \\
\hline no 193 & Extremamente preocupado & Sim & $<0,048$ \\
\hline
\end{tabular}

Produzido pelos autores. Nota: $* 193-$ Subir ou descer escadas.

em questões extradomiciliares e se manifestou muito ou extremamente preocupado em cair. Em um estudo realizado por Keal e colaboradores, os autores concluem que idosos que apresentaram medo de cair em ambientes externos e sociais apresentam-se mais capazes em termos de status psicossocial, físico e funcional quando comparados a idosos que apresentam o medo de cair exclusivamente em ambiente doméstico, uma vez que essas atividades exigem um maior controle do equilíbrioe manutenção da estabilidade. ${ }^{19}$ Estes autores afirmam que idosos com "medo de cair" evitam situações desafiadoras e acabam restringindo-se ao ambiente domiciliar.

Dados obtidos em diferentes pesquisas mostram que o medo de cair está presente em atividades que podem também ser executadas dentro de casa, como subir e descer escadas e andar em superfícies escorregadias, constituindo-se como fatores de risco/ causa para o evento de queda. ${ }^{20,21} \mathrm{Em}$ contrapartida, se esses idosos se restringem ao ambiente domiciliar, o número de quedas será maior dentro de casa, uma vez que as causas estão relacionadas a fatores extrínsecos, como escadas e superfícies escorregadias. ${ }^{20}$ No presente estudo os resultados mais significativos nos dois grupos (GDAM e GSDAM) foram relacionados às características do ambiente físico. Por se tratar de uma amostra com características de fragilidade, é possível que estes idosos passem a maior parte de seu tempo no domicílio, sendo de relevante importância estratégias de prevenção dos eventos de quedas neste ambiente com possibilidades de adaptações nos locais de risco. ${ }^{22}$

Alguns estudos apontam que o conjunto de elementos que geram risco para quedas e a ocorrência prévia do evento podem ser considerados como um dos principais fatores de risco para a diminuição da autoeficácia. ${ }^{810}$ Diversos estudos indicam também que atividades como subir ou descer escadas, tomar banho, caminhar sobre superfície irregular e andar sobre uma superfície escorregadia são atividades que apresentam alto grau de exigência do sistema osteomioarticular e de equilíbrio. Neste contexto, a utilização do DAM associado a tais situações pode levar a quedas.22,23
Observamos que $44 \%$ dos idosos que usam DAM sofreram eventos de quedas comparados a $34 \%$ dos que não usam. Nesta perspectiva, vale ressaltar a importância da investigação quanto ao ambiente da queda e o tipo de atividade realizada para que seja possível um planejamento de intervenções bem direcionadas para prevenção de novos eventos, considerando que grande parte dos idosos acredita que o uso do DAM não énecessário no ambiente doméstico, utilizando paredes e móveis como apoio. ${ }^{24}$

Conclui-se que o uso de DAM não necessariamente aumenta a autoeficácia para quedas em idosos, não devendo por isso ser considerado como um meio único e confiável na prevenção deste evento. Desta forma, é fundamental a identificação de fatores de risco para quedas associadas ao uso de DAM, que indiquem sua ocorrência para possibilitar o manejo e a intervenção em situações de risco, tendo como foco a prevenção e o cuidado em saúde.

\section{Referências}

1. Brasil. Ministério da Saúde. Secretaria de Atenção à Saúde. Departamento de Atenção Básica. Envelhecimento e saúde da pessoa idosa. Brasília: Ministério da Saúde; 2010.

2. Pereira GN, Morsch P, Lopes DGC, et al. Fatores socioambientais associados à ocorrência de quedas em idosos. Ciênc Saúde Coletiva. 2013;18(12):3507-3514.

3. Glisoi SFN, Ansai JH, da Silva TO, et al. Dispositivos auxiliares de marcha: orientação quanto ao uso, adequação e prevenção de quedas em idosos. SBGG. 2012;6(3):261-272 [acesso em 28 mai 2017]. Disponível em: http://sbgg.org.br/wp-content/ uploads/2014/10/2012-3.pdf

4. Rico NC, Lemos ND, Ferreira CV, et al. Uso de dispositivo de auxílio à marcha: a percepção dos idosos. Rev Equilíbrio Corpor e Saúde. 2012;4(2):42-50.

5. Bradley SM, Hernandez CR. Geriatric assistive devices. Am Fam Physician. 2011;84(4):405-11.

6. Cruz DT da, Ribeiro LC, Vieira M de T, et al. Prevalência de quedas e fatores associados em idosos. Rev Saude Publica. 2012;46(1):138-46.

7. Rodrigues IG, Fraga GP, Barros MB de A. Falls among the elderly: risk factors in a population-based study. Rev Bras Epidemiol. 2014;17(3):705-18.

8. Klima D, Newton R, Keshner E, et al. Fear of falling and balance ability in older men: the priest study. J Aging Phys Act. 2013;21(4):375-86. 


\section{Artigo original}

9. Cruz DT, Duque RO, Leite ICG. Prevalência do medo de cair em uma população de idosos da comunidade. Rev Bras Geriatr Gerontol. 2017;20(3):309-318.

10. Dias RC, Freire MTF, Santos EGS, et al. Características associadas à restrição de atividades por medo de cair em idosos comunitários. Rev Bras Fisioter. 2011;15(5):406-13.

11. Lourenco RA, Moreira VLG, Banhato EFC, et al. Prevalence of frailty and associated factors in a community- dwelling older people cohort living in Juiz de Fora, Minas Gerais, Brazil: Fibra-JF Study. Ciênc Saúde Coletiva. 2019;24(1):35-44.

12. Yardley L, Beyer N, Hauer K, et al. Development and initial validation of the Falls Efficacy Scale-International (FES-I). Age Ageing. 2005;34(6):614-9.

13. Camargos FFO, Dias RC, Dias JMD, et al. Adaptação transcultural e avaliação das propriedades psicométricas da Falls Efficacy Scale - International em idosos Brasileiros (FES-I-BRASIL). Rev Bras Fisioter. 2010;14(3):237-243.

14. Soares DS, Jefferson W, Moraes A De, et al. Fatores associados a quedas e quedas recorrentes em idosos: estudo de base populacional. Rev Bras Geriatr e Gerontol. 2014;17(1):49-60.

15. Antes DL, D'Orsi E, Benedetti TRB. Circunstâncias e consequências das quedas em idosos de Florianópolis. Epifloripa Idoso 2009. Rev Bras Epidemiol. 2013;16(569834):469-81.

16. Gaxatte $C$, Nguyen T, Chourabi F, et al. Fear of falling as seen in the Multidisciplinary falls consultation. Ann Phys Rehabil Med. 2011;54(4):248-58.

17. Falsarella GR, Gasparotto LPV, Coimbra AMV. Quedas: conceitos, frequências e aplicações à assistência ao idoso. Revisão de literatura. Rev Bras Geriatr Gerontol. 2014;17(4):897-910.

18. Kumar A, Carpenter $\mathrm{H}$, Morris $\mathrm{R}$, et al. Which factors are associated with fear of falling in community-dwelling older people? Age Ageing. 2014;43(1):76-84.

19. Keall M.D, Pierse N, Howden-Chapman P, et al. Home modifications to reduce injuries from falls in the Home Injury Prevention (HIPI) study: a cluster-randomised controlled trial. Lancet. 2015;385(9964):231-38.

20. Nicolussi AC, Fhon JRS, Santos CAV, et al. Qualidade de vida em idosos que sofreram uma queda: revisão integrativa da literatura. Ciênc Saúde Coletiva. 2012;17(3):723-730.

21. Fucahori FS, Lopes AR, Correia JJA, et al. Fear of falling and activity restriction in older adults from the urban community of Londrina: a cross-sectional study. Fisioter em Mov [Internet]. 2014;27(3):379-87. Disponível em: http://www.scielo.br/scielo.php?script=sci_arttext\&pi$d=$ S0103-51502014000300379\&lng=en\&tIng=en

22. Goodwin V, Jones-Hughes T, Thompson-Conn J, et al. Implementing the evidence for preventing falls among community dwelling older people: A systematic review. J Safety Res. 2011;42:443-51.

23. Freitas R, Santos SSC, Hammerschmidt KSA, et al. Cuidado de enfermagem para prevenção de quedas em idosos: proposta para ação. Rev Bras Enferm. 2011 Jun;64(3):478-485.

24. Sarmento A, Fernandes P, Bestetti M, et al. Fatores ambientais e risco de quedas em idosos: revisão sistemática. Rev Bras Geriatr Gerontol. 2014;17(3):637-645 